\title{
Transcranial Magnetic Stimulation-Derived Motor Parameters and Regional Glucose Metabolism of Motor Cortex
}

\author{
Cheng-Ta $\mathrm{Li}^{1-3}$, Chi-Hung Juan ${ }^{3}$, Tung-Ping Su ${ }^{1,2,4}$, Ying-Jay Liou ${ }^{1,2}$, Mu-Hong Chen ${ }^{1,2}$, Chih-Ming Cheng ${ }^{1}$ and Ying-Zu Huang ${ }^{3,5 *}$ \\ ${ }^{1}$ Department of Psychiatry, Taipei Veterans General Hospital, Taipei, Taiwan \\ ${ }^{2}$ Division of Psychiatry, Faculty of Medicine, National Yang-Ming University, Taipei, Taiwan \\ ${ }^{3}$ Institute of Cognitive Neuroscience, National Central University, Jhongli, Taiwan \\ ${ }^{4}$ Institute of Brain Science, National Yang-Ming University, Taipei, Taiwan \\ ${ }^{5}$ Neuroscience Research Center and Department of Neurology, Chang-Gung Memorial Hospital and Chang-Gung University College of Medicine, Taoyuan, Taiwan
}

\begin{abstract}
Objective: Transcranial Magnetic Stimulation (TMS) has become a widely used and excellent method for examining cortical excitability abnormalities in the human system. However, the direct link between TMS measurements and its representation of regional neural brain activity is yet to be determined. We hypothesize that TMS measurements we do hypothesize that TMS parameter measurements of the motor cortices of both the dominant-side and non-dominant side would reflect direct correlations with their respective regional neural activities of the targeted motor cortex.
\end{abstract}

Methods: Twenty-Seven right-handed subjects were recruited under the Edinburgh Handedness Inventory. All subjects were healthy, and were exposed to excitatory and inhibitory TMS pulses from the bilateral motor cortices and brain glucose metabolism. Brain glucose metabolism was measured by ${ }^{18} \mathrm{~F}$-fluorodeoxyglucose positron emission tomography $\left({ }^{18} \mathrm{~F}-\mathrm{FDG}-\mathrm{PET}\right)$ at rest.

Results: All procedures were well tolerated with no adverse events. The voxel-wise analysis demonstrated significant relationship between the dominant-side TMS parameters (including resting motor threshold, motor evoked potentials (MEP) amplitude, along with long interval intra-cortical inhibition) with the dominant-side motor related areas (family-wise errors-corrected $p<0.005$ ). In contrast to this finding, our study found that TMS measurements from the non-dominant side were connected to areas beyond the non-dominant-side motor areas in both hemispheres.

Conclusion: TMS parameters from the dominant-side motor cortices better represent target motor cortex and their regional neural activities.

Significance: This study finds variation in stimulation parameters measured from the different bilateral motor cortices.

Keywords: Motor cortex; Cortical excitability; Transcranial magnetic stimulation; Intracortical inhibition; Handedness

\section{Introduction}

Transcranial magnetic stimulation (TMS) is a non-invasive brain stimulation technique used in assessing cortical excitability. Barker et al. first introduced TMS to examine motor pathways in 1985 [1] and found that a single TMS pulse was able to stimulate the motor cortex causing contractions in the muscles of the associated hand or leg muscles to elicit motor evoked potentials (MEPs).

TMS has become an exceptional technique for studying the abnormalities of cortical excitability associated with major neuropsychiatric disorders due to its ability in detecting subtle deficits in brain inhibition and excitation [2-4]. The TMS tool can measure resting motor threshold (rMT) and the amplitude of motor evoked potentials (MEPs) using single-pulse TMS (spTMS), intra-cortical facilitation (ICF), and paired-pulse TMS (ppTMS) to examine cortical excitability. Inhibitory circuits, such as short-interval cortical inhibition (SICI) and long-interval cortical inhibition (LICI), can be measured using a spTMS-induced silent period and ppTMS paradigms [2-4]. Despite methodology variations in previous studies, the TMS method has been providing informative results in the study of neuropsychiatric disorders [4].

Determining which motor cortex sides (i.e., dominant or nondominant hemisphere) should be tested and whether TMS results measured from either side mean the same thing have not yet been determined. The dominant hemisphere is frequently studied more as it is believed to be a genuine representative of the entire brain Nevertheless, the non-dominant hemisphere is also important. A vast majority of previous studies have focused on cortical excitatory and inhibitory deficits of the left hemisphere (dominant side in right-handed subjects) [5-9]. Occasional cortical excitability of the right hemisphere have however been reported in previous studies that focused on both hemispheres [10-12], which may be the explanation for most studies adhering to the non-dominant hemisphere only [13].

The motor cortex is the center for controlling and modulating motor action which relies on a sensitive balance between cortical excitatory and inhibitory mechanisms. TMS pulses indirectly activate pyramidal neurons via synaptic inputs, which subsequently induce efferent volleys in the corticospinal tract. This efferent volley occurs as a result of the TMS-induced electrical current which generally activates horizontally oriented neurons rather than vertically oriented

*Corresponding author: Cheng-Ta Li, Department of Psychiatry, Taipei Veterans General Hospital, Taiwan and No. 201, Section 2, Shih-Pai Road, Beitou District, Taipei 112, Taiwan, Tel: +886-2-28757027; Ext: 298; Fax: +886-2-28757592; E-mail: ctli2@vghtpe.gov.tw

Received July 13, 2016; Accepted July 25, 2016 ; Published July 29, 2016

Citation: Li CT, Juan CH, Su TP, Liou YJ, Chen MH, et al. (2016) Transcrania Magnetic Stimulation-Derived Motor Parameters and Regional Glucose Metabolism of Motor Cortex. J Neurol Neurophysiol 7: 386. doi:10.4172/2155-9562.1000386

Copyright: @ 2016 Li CT, et al. This is an open-access article distributed under the terms of the Creative Commons Attribution License, which permits unrestricted use, distribution, and reproduction in any medium, provided the original author and source are credited. 
pyramidal neurons. Electrical currents from TMS pulses evoke action potential confined to the local neural cell population which may either be inhibitory or excitatory [14]. Motor parameters from both singlepulse and paired-pulse TMS signals are believed to reflect inhibitory and excitatory functions of the target motor cortex [15-17]. However, the mechanism of TMS-derived motor parameters and its relationship to neural activities internal and external to the motor cortex is not completely understood.

Our study targeted to exploit the relationships between brain neural activities and TMS measurements of excitation and inhibition in both the dominant and non-dominant motor cortices. ${ }^{18} \mathrm{~F}$-fluorodeoxyglucose positron emission tomography $\left({ }^{18} \mathrm{~F}-\mathrm{FDG}\right.$-PET) was used to estimate neural activity as it contains a mixture of glutamatergic excitatory and GABAergic inhibitory neural component which parallel the underlying mechanism of TMS-measured excitation and inhibition [15-17]. Secretions and uptakes of glutamate and GABA in the neuronal synapses are closely related to glucose utilization in the neurons and astrocytes [18]. ${ }^{18} \mathrm{~F}-\mathrm{FDG}$-PET signaling is widely used as a relatively specific proxy for measuring glutamatergic neurotransmission in refractory depressive patients $[19,20]$. On the contrary, ${ }^{18} \mathrm{~F}-\mathrm{FDG}$ changes in the brain may also reflect GABA-related inhibitory functions. Consistent with previous studies that examined antidepressant treatment in rodents, it was found that increased striatal ${ }^{18} \mathrm{~F}$-FDG uptake and GABA (but not glutamate) was related to antidepressants after four weeks of treatment [21].

The present study investigated TMS measurements on the bilateral motor cortex in a resting state and its subsequent relationships with regional ${ }^{18} \mathrm{~F}$-FDG uptakes in the brain. All subjects are healthy and right-handed. We hypothesized that TMS parameters measured from the dominant-side and the non-dominant side of motor cortex may correlate with the regional neural activities of the dominant-side and the non-dominant side motor cortex, respectively.

\section{Methods}

\section{Subjects}

All 27 right-handed subjects were healthy and had no history of neurological illness, substance and alcohol abuse (mean age, $36.4 \pm 10.6$ years, ranging from 22 to 60 years, with 14 females). No participants were on any medications during experiment exposure. Participants also did not have psychiatric disorders of any sort (concluded using the Mini International Neuropsychiatric Interview (MINI) using the Diagnostic and Statistical Manual of Mental Disorders-IV-Text Revision (DSMIV-TR) criteria (American Psychiatric Association, 2000). Handedness among subjects was confirmed using the Edinburgh Handedness Inventory [22]. Approval from the Ethic's Review Committee of Taipei Veteran's General Hospital and the Declaration of Helsinki was maintained throughout the study. Informed consents were provided by all study participants before conducting experiments.

\section{Single-pulse and paired-pulse TMS procedures}

$\mathrm{Ag}-\mathrm{AgCl}$ electrodes were positioned with the active electrode over the abductor Pollicis brevis (APB) belly. Surface electromyography (EMG) recordings from the APB were recorded.

All participants maintained a relaxed state throughout the study. 70 $\mathrm{mm}$ figure-of-eight coil were connected to two Magstim 200 magnetic stimulators through a bi-stim module (Magstim Company. Whitland UK) in two separate sessions. Coils were placed with heads secured tangentially in position ensuring coil handles pointing backwards approximately 45 degrees distal from the mid-sagittal line for eliciting MEPs corresponding to the left or right APB muscles.

Maintaining this coil position, the induced currents via transsynapses from the brain predominantly activates the cortocospinal fibers. The best suited positions were marked directly on the scalps of participants ensuring identical coil positioning throughout the study.

Resting motor threshold (rMT) was taken as the lowest intensity; expressed as a percentage of the maximum stimulator output. rMT in this study emitted a MEP of $\pm 50 \mu \mathrm{V}$ from a total of five to ten trials in the relaxed APB muscle of participants [23].

Intra-cortical facilitation (ICF) and short-interval intra-cortical inhibition (SICI) were analyzed with a subthreshold conditioning stimulus (CS) of $80 \%$ rMT preceded by a suprathreshold testing stimulus (TS) of $120 \%$ rMT (Kujirai et al., 1993). Interstimulus intervals (ISIs) between CS and TS were examined at 2,10 and $20 \mathrm{~ms}$ (CS2, CS10, CS20).This study evaluated long-interval intra-cortical inhibition (LICI) using suprathreshold CS followed by TS of $120 \%$ rMT at ISIs of 100 and $200 \mathrm{~ms}$ (CS100 and CS200) [24].

As numerous studies previously adopted five to ten trials for each ISI [10-12], each session for a single side of the motor cortex in the present study adopted eight trials of just TS (unconditioned responses). Similarly, eight trials were also used of the five conditioned stimuli (i.e., CS2, CS10, CS20, CS100, and CS200).

In total, 48 trials were performed every eight seconds in random sequence. Signal (Version 6.02, Cambridge Electronic Design Ltd. Cambridge, England) was used to program to automatically adjust all trials and their stimulus intensities through a control cable. The order of the two sessions was alternated among subjects to strategically minimize potential order effects. The conditioned MEPs were represented as a ratio of the mean MEP amplitude (ratio of the conditioned response and the unconditioned response). SICI estimation was defined as the conditioned MEP amplitude of CS2. ICF estimation and LICI was elucidated with the mean of the conditioned MEP amplitudes of CS10 and CS20, and CS100 and CS200, respectively.

\section{Measurements of cortical excitation and inhibition}

Cortical excitability parameters included the rMT, the mean MEP amplitude (120\% rMT using solely TS), and ICF. For cortical inhibition, SICI and LICI were taken as the parameters.

\section{Obtaining positron emission tomography (PET) data}

PET scans of glucose utilization were obtained using a PET/CT scanner in 3D brain mode (Discovery VCT; GE Healthcare, USA) [25].

Participants were required to fast for a period of 8 hours before the PET scans were performed.

After 45 min following an intravenous injection, PET images were obtained at about $370 \mathrm{MBq}$ of ${ }^{18} \mathrm{~F}$-FDG. The transaxial resolution fullwidth at half-maximum (FWHM) was $5.12 \mathrm{~mm}(1 \mathrm{~cm}$ off-center $)$ and the axial resolution was $5.18 \mathrm{~mm}$.

Brain acquisition time was 15 min during which 47 consecutive slices were created over an axial length of $15.7 \mathrm{~cm}$; with $3.75 \mathrm{~mm}$ slice thickness and $70 \mathrm{~cm}$ transaxial FOV. Reconstruction of the images obtained was performed in a $128 \times 128$ matrix with attenuation corrections using CT information alongside ordered-subset expectation maximization (OS-EM) reconstruction algorithms (6 iterations and 14 subsets). 
Axial images were realigned producing sagittal and coronal images. The counts in each image were converted to radioactivity concentration using calibration factors obtained from a cylinder phantom scan of ${ }^{18} \mathrm{~F}-\mathrm{FDG}$ and phantom activity aliquot.

All images were then normalized by injected dose and body weight to obtain standardized uptake value images [26]. All PET scans were taken on the same day TMS were taken.

\section{Voxel-wise analysis of PET data}

Statistical Parametric Mapping version 8 software (SPM8; Wellcome Department of Cognitive Neurology, Institute of Neurology, University College London, London, England) was used for data analysis as implemented in Matlab 7.1 (The Mathworks Inc., Sherborn, MA, USA) to analyze the PET data. Each subject's PET images were normalized to standard MNI brain space $[27,28]$ and smoothed using 3D Gaussian kernel (full width half maximum [FWHM] $=8 \mathrm{~mm}$ ). The resulting images in the standardized brain space were then subjected to further analysis.

Voxel-based partial correlation was analyzed separately, controlling age, gender, and global glucose uptakes, to determine presence of relationship between the PET images and TMS parameters of cortical excitation and inhibition (i.e., rMT, MEP amplitude, ICF, SICI and LICI).

For the a-priori region-'motor cortex', voxels passing uncorrected at $\mathrm{p}<0.005$ were used and subsequently examined using small volume correction (SVC) comprising of an anatomically defined regional mask in the relevant gray matter area. Threshold significance was strictly set at cluster level family-wise errors (FWE) corrected $\mathrm{p}<0.005$ where the FWE was used to correct multiple comparisons) for all other brain regions. Brain regions that exceeded the statistical threshold in the correlation analysis were considered the most significant brain regions related to the TMS parameters.

PMOD version 3.0 (PMOD Technologies, Zurich, Switzerland) was used in determining the mean brain glucose uptake of the motor cortex from the unsmoothed PET images in the standard stereotactic space, as previously described [29]. The normalized glucose metabolism of the motor cortex was standardized by dividing the uptake values of the motor cortex by the global mean uptake values was also reported.

\section{Statistics for TMS parameters}

Using SPSS 16.0 software (SPSS Inc., Chicago, IL), the paired $t$-test was applied to compare the continuous variables of all TMS parameters and the brain regional glucose metabolism between the dominant and non-dominant hemisphere of all participants. An independent $t$-test was used to compare the continuous variables between males and females. To be statistically significant, Statistical significance was determined with a p-value $<0.05$.

\section{Results}

Concluding from all the TMS measurements, only LICI differed considerably between the dominant (left) and non-dominant (right) sides (Figure 1). Non-dominant-side LICI $(0.39 \pm 0.22)$ was significantly greater than dominant-side LICI $(0.54 \pm 0.28)(\mathrm{t}=2.640, \mathrm{p}=0.013)$. The normalized regional glucose metabolism (rGluM) of the dominant motor cortex $(1.101 \pm 0.019)$ was found to be significantly greater than that of the non-dominant motor cortex $(1.081 \pm 0.022, t=6.207$, $\mathrm{p}<0.001)$. None of the measurements revealed differences between genders (all $\mathrm{p}>0.05$ ).

The voxel-wise associations between rGluM and TMS-measured excitation and inhibition are shown in Tables 1 and 2, respectively.

TMS measurements from the dominant left hemisphere demonstrated that a greater rMT value corresponded to a greater rGluM value in the dominant-side of the motor cortex (Brodmann area 4, BA4), premotor cortex (BA6), and middle frontal cortex (BA8) (Table 1 , which shows all clusters that exceeded statistical threshold with peak voxels in each identified cluster). However, TMS measurements from the non-dominant right motor cortex (M1) showed a significant correlation between rMT and rGluM in a minor section of the left motor cortex, but various parts of the bilateral frontal cortices (BA9 and BA11) shown in Table 1.

The mean MEP amplitude from the dominant left hemisphere demonstrated a negative correlation with rGluM in the dominant motor cortex (BA4), the left postcentral gyrus (BA3, parts of sensory cortex), and the left inferior parietal gyrus (BA40).

The mean MEPs amplitude of the non-dominant right hemisphere showed a widespread negative association with the bilateral motor cortices (BA4), postcentral gyri (BA1, parts of sensory cortex), middle
(A)

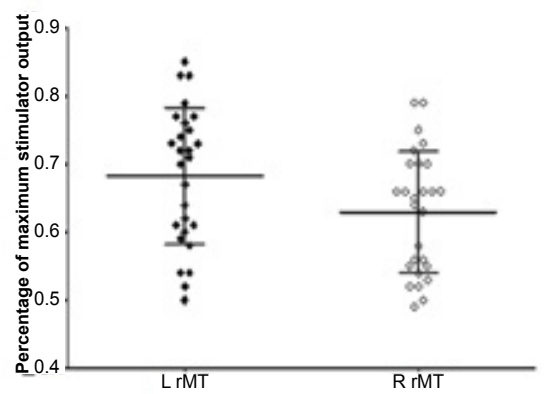

(B)

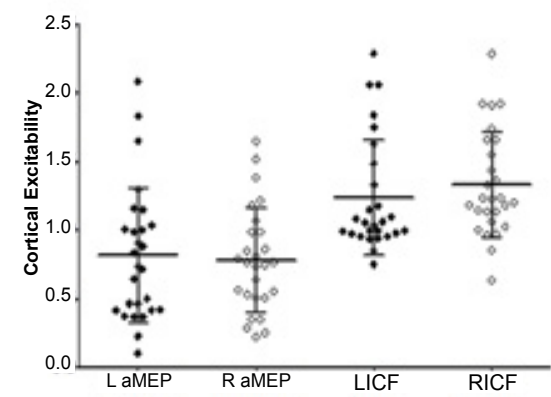

(C)

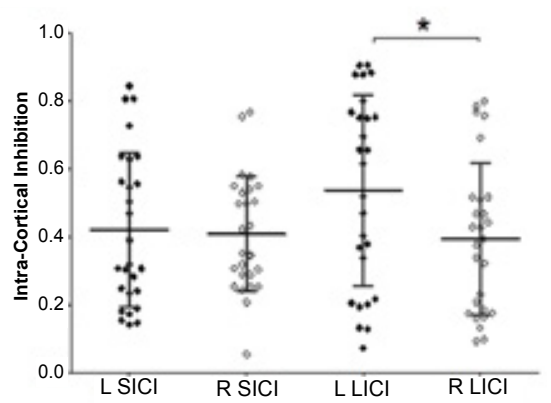

${ }^{*} p<0.05$

Figure 1: Side-to-side comparisons of cortical excitation and inhibition in healthy right-handed patients. (A) Left-side (dominant-side) rMT (68.2 +/- 10\%) was not significantly different from right-side rMT $(64.2+/-10.1 \%)$. (B) The measurements of cortical excitation did not differ between the dominant (left) and non-dominant (right) hemispheres. (C) The majority of the measurements of cortical inhibition did not differ between the left and right hemispheres, except for long-interval intracortical inhibition. L, Left; R, Light; rMT, Resting Motor Threshold; aMEP, Amplitude of Motor Evoked Potentials; ICF, Intra-Cortical Inhibitions; SICI, Short-Interval Intra-Cortical Inhibition; LICI, Long-Interval Intra-Cortical Inhibition; NS, No Statistical Significance; A comparison of the left and right-side values in all the recruited participants by paired $t$-tests. 
Citation: Li CT, Juan CH, Su TP, Liou YJ, Chen MH, et al. (2016) Transcranial Magnetic Stimulation-Derived Motor Parameters and Regional Glucose Metabolism of Motor Cortex. J Neurol Neurophysiol 7: 386. doi:10.4172/2155-9562.1000386

Page 4 of 8

\begin{tabular}{|c|c|c|c|c|c|c|c|c|}
\hline \multirow{2}{*}{ Groups } & \multirow{2}{*}{ Anatomical regions } & \multirow{2}{*}{ Cluster size } & \multicolumn{3}{|c|}{ MNI coordinates } & \multirow{2}{*}{$\mathbf{t}$} & \multirow{2}{*}{$\mathbf{z}$} & \multirow{2}{*}{ p-value } \\
\hline & & & $\mathbf{x}$ & $\mathbf{y}$ & $\mathbf{z}$ & & & \\
\hline \multicolumn{9}{|l|}{ rMT: Dominant (Left) } \\
\hline \multirow[t]{3}{*}{ Positive } & L Premotor Area (BA 6) & 1384 & -48 & 6 & 46 & 4.47 & 3.68 & 0.001 \\
\hline & L Middle Frontal Gyrus (BA 8) & & -50 & 18 & 42 & 3.83 & 3.36 & \\
\hline & L Motor Cortex (BA 4) & & -40 & -16 & 54 & 3.27 & 2.86 & \\
\hline Negative & - & - & - & - & - & - & - & - \\
\hline \multicolumn{9}{|l|}{ rMT: Non-Dominant (Right) } \\
\hline \multirow[t]{5}{*}{ Positive } & L Motor cortex (BA 4) & 1883 & -46 & -2 & 60 & 3.85 & 3.25 & 0.002 \\
\hline & L Superior Frontal Gyrus (BA9) & & -6 & 52 & 36 & 3.85 & 3.25 & \\
\hline & L Orbitofrontal Gyrus (BA 11) & 2637 & -14 & 46 & -14 & 4.83 & 3.82 & 0.001 \\
\hline & R Orbitofrontal Gyrus (BA 11) & & 26 & -12 & -14 & 3.97 & 3.32 & \\
\hline & R Superior Frontal Gyrus (BA9) & & 8 & 54 & 42 & 3.41 & 2.96 & \\
\hline Negative & - & - & - & - & - & - & - & - \\
\hline \multicolumn{9}{|l|}{ MEP amplitude: Dominant (Left) } \\
\hline Positive & - & - & - & - & - & - & - & - \\
\hline \multirow[t]{3}{*}{ Negative } & L Motor Cortex (BA 4) & 1204 & -40 & -14 & 34 & 3.52 & 3.03 & 0.003 \\
\hline & L Inferior Parietal Gyrus (BA 40) & & -52 & -42 & 58 & 3.24 & 2.84 & \\
\hline & L Postcentral Gyrus (BA 3) & & -24 & -38 & 58 & 3.01 & 2.68 & \\
\hline \multicolumn{9}{|c|}{ MEP amplitude: Non-Dominant (Right) } \\
\hline Positive & - & - & - & - & - & - & - & - \\
\hline \multirow[t]{12}{*}{ Negative } & L Postcentral Gyrus (BA 1) & 9517 & -22 & -28 & 80 & 5.46 & 4.14 & 0 \\
\hline & L Motor Cortex (BA 4) & & -40 & -14 & 50 & 5.1 & 3.96 & \\
\hline & L Superior Parietal Lobule (BA 7) & & -16 & -68 & 68 & 4.68 & 3.74 & \\
\hline & L Middle Occipital Gyrus (BA 18) & & -16 & -98 & 10 & 4.26 & 3.49 & \\
\hline & L Middle Frontal Gyrus (BA 8) & & -24 & 30 & 52 & 3.02 & 2.72 & \\
\hline & L Superior Temporal Gyrus (BA 41) & & -50 & -40 & 10 & 3.18 & 2.8 & \\
\hline & R Superior Temporal Gyrus (BA 41) & & 46 & -40 & 12 & 4.68 & 3.74 & \\
\hline & R Middle Frontal Gyrus (BA 8) & & 24 & 26 & 50 & 3.37 & 2.93 & \\
\hline & R Postcentral Gyrus (BA 1) & & 18 & -44 & 66 & 2.91 & 2.6 & \\
\hline & R Motor Cortex (BA 4) & & 24 & -30 & 68 & 2.88 & 2.58 & \\
\hline & R Middle Occipital Gyrus (BA 19) & & 48 & -82 & 26 & 3.03 & 2.69 & \\
\hline & Cingulate Gyrus (BA 24) & & 0 & 20 & 32 & 3.12 & 2.75 & \\
\hline \multicolumn{9}{|c|}{ Intra-cortical Facilitation: Dominant (Left) } \\
\hline Positive & - & - & - & - & - & - & - & - \\
\hline Negative & - & - & - & - & - & - & - & - \\
\hline \multicolumn{9}{|c|}{ Intra-cortical Facilitation: Non-Dominant (Right) } \\
\hline \multirow[t]{8}{*}{ Positive } & L Postcentral Gyrus (BA 2) & 9111 & -26 & -46 & 68 & 5.4 & 4.11 & 0 \\
\hline & L Paracentral Lobule (BA5) & & -18 & -44 & 52 & 5.17 & 4 & \\
\hline & L Motor Cortex (BA 4) & & -52 & -12 & 44 & 4.42 & 3.59 & \\
\hline & L Middle Frontal Gyrus (BA 8) & & -48 & 10 & 42 & 3.59 & 3.08 & \\
\hline & R Inferior Parietal Lobule (BA 40) & & 46 & -52 & 58 & 4.31 & 3.53 & \\
\hline & Cingulate Gyrus (BA 24) & & 10 & -8 & 32 & 3.78 & 3.2 & \\
\hline & R Postcentral Gyrus (BA 3) & & 36 & -40 & 62 & 3.1 & 2.74 & \\
\hline & Supplementary Motor cortex (BA 6) & & 0 & -20 & 54 & 3.65 & 3.12 & \\
\hline Negative & - & - & - & - & - & - & - & - \\
\hline
\end{tabular}

(L-Left; R-Right; Positive-Positive correlation; Negative-Negative correlation; rMT-Resting Motor Threshold; MEP-Motor Evoked Potentials. Except for the a-priori regionmotor cortex (Precentral Gyrus), all reported $p$-values passed FWE-corrected $p<0.005$

Table 1: Correlation of cortical excitation and regional glucose metabolism.

occipital gyri (BA19), middle frontal gyri (BA8), superior temporal gyri (BA 41) and middle cingulate gyri (BA 24) shown in Table 1.

No correlation was found between the ICF and SICI measured from the dominant hemisphere and rGluM. A positive correlation was found between ICF from the non-dominant right $\mathrm{M} 1$ and rGluM in the left motor cortex (BA4), left paracentral lobule (BA5), left middle frontal gyrus
(BA8), bilateral postcentral gyri (BA2 and BA4), bilateral supplementary motor cortices (BA6) and cingulate gyri (BA24) shown in Table 1.

There was a negative correlation found between the non-dominantside SICI values and rGluM of the bilateral thalami, putamen, inferior frontal gyri, and the right prefrontal cortex (BA 9 and 46) demonstrated in Table 2. 


\begin{tabular}{|c|c|c|c|c|c|c|c|c|}
\hline \multirow{2}{*}{ Groups } & \multirow{2}{*}{ Anatomical regions } & \multirow{2}{*}{$\begin{array}{c}\text { Cluster } \\
\text { size }\end{array}$} & \multicolumn{3}{|c|}{ MNI coordinates } & \multirow{2}{*}{$\mathbf{t}$} & \multirow{2}{*}{$\mathbf{Z}$} & \multirow{2}{*}{ p-value } \\
\hline & & & $\mathbf{x}$ & y & z & & & \\
\hline \multicolumn{9}{|c|}{ Short-interval intra-cortical inhibition: Dominant (Left) } \\
\hline Positive & - & - & - & - & - & - & - & - \\
\hline Negative & - & - & - & - & - & - & - & - \\
\hline \multicolumn{9}{|c|}{ Short-interval intra-cortical inhibition: Non-Dominant (Right) } \\
\hline Positive & - & - & - & - & - & - & - & - \\
\hline \multirow[t]{8}{*}{ Negative } & L Thalamus & 8990 & -8 & -20 & 10 & 3.68 & 3.14 & 0.000 \\
\hline & L Inferior Frontal Gyrus & & -30 & 28 & -18 & 3.75 & 3.18 & \\
\hline & L Putamen & & -30 & 12 & 6 & 3.28 & 2.86 & \\
\hline & R Prefrontal Cortex (BA 46) & & 46 & 44 & 10 & 3.32 & 2.9 & \\
\hline & R Prefrontal Cortex (BA 9) & & 32 & 34 & 28 & 3.15 & 2.78 & \\
\hline & R Putamen & & 20 & 12 & 8 & 4.48 & 3.63 & \\
\hline & R Thalamus & & 24 & -32 & 4 & 3.92 & 3.29 & \\
\hline & R Inferior Frontal Gyrus & & 36 & 24 & -18 & 3.66 & 3.12 & \\
\hline \multicolumn{9}{|c|}{ Long-interval intra-cortical inhibition: Dominant (Left) } \\
\hline \multirow[t]{3}{*}{ Positive } & L Inferior Parietal Lobule (BA40) & 1204 & -38 & -52 & 66 & 5.57 & 4.19 & 0.001 \\
\hline & L Postcentral Gyrus (BA3) & & -56 & -22 & 36 & 4.02 & 3.35 & \\
\hline & L Motor Cortex (BA 4) & & -40 & -26 & 62 & 3.52 & 3.03 & \\
\hline Negative & - & - & - & - & - & - & - & - \\
\hline \multicolumn{9}{|c|}{ Long-interval intra-cortical inhibition: Non-Dominant (Right) } \\
\hline \multirow[t]{7}{*}{ Positive } & L Motor Cortex (BA 4) & 927 & -34 & -24 & 60 & 3.79 & 3.21 & 0.002 \\
\hline & L Caudate & & -10 & 22 & -2 & 3.05 & 2.70 & \\
\hline & L Postcentral Gyrus (BA 3) & & -44 & -22 & 52 & 3.02 & 2.68 & \\
\hline & R Motor Cortex (BA 4) & 676 & 40 & -8 & 54 & 4.58 & 3.68 & \\
\hline & R Putamen & & 34 & 4 & 8 & 3.39 & 2.94 & \\
\hline & R Postcentral Gyrus (BA 3) & & 30 & -26 & 56 & 3.05 & 2.70 & \\
\hline & R Caudate & & 6 & 22 & -2 & 3.02 & 2.68 & \\
\hline Negative & - & & & & & & & \\
\hline
\end{tabular}

(L-Left; R-Right; Positive-Positive correlation; Negative-Negative correlation; rMT-Resting Motor Threshold; MEP-Motor Evoked Potentials. Except for the a-priori region'motor cortex', all reported p-values passed FWE-corrected $\mathrm{p}<0.005)$

Table 2: Correlation of cortical inhibition and regional glucose metabolism.

The dominant-side left LICI showed positive correlation with rGluM from the dominant-side motor cortex (BA4), left postcentral gyrus (BA3), and left inferior parietal lobule (BA40). On the contrary, the non-dominant right LICI showed positive correlation with the bilateral motor cortices (BA4), caudates and post central gyri (BA3) summarized in Table 2 .

Concluding from these results, we found that the correlations between whole-brain glucose metabolism and the dominant-side TMS parameters from cortical excitation and inhibition (including $\mathrm{rMT}$ (Figure 2A1), MEP amplitude (Figure 2B1), and LICI (Figure 2C1)) were found to be more focused in the dominant (left) motor cortex and its nearby cortical regions. On the other hand, the correlations from non-dominant right-side TMS parameters were found to be more widespread and bilaterally located (Figures $2 \mathrm{~A} 2$ and 2C2).

\section{Discussion}

This study has provided novel conclusion that TMS derived motor parameters from the bilateral motor cortices show variations in measurements with brain neural parameters and possibly elucidating variety of implications. TMS measurements taken from the dominant (left) motor cortex are related mainly to rGluM within the left motor area, which is indicative that the TMS-derived parameters from the dominant motor cortex is indeed a better reflection of regional neural activities of the targeted dominant-side motor cortex. However, the relationship between TMS measurements from the non-dominant side and rGluM were relatively widespread across areas over both hemispheres. Considering a higher influence of the non-dominant side of the motor cortex by other brain regions, they may have the potential to reflect cortical inhibitory and excitatory abnormalities outside of the motor cortex which should captivate and focus future research attention to the non-dominant side.

This study found that TMS parameters from the dominant motor cortex, unlike parameters from the non-dominant side, were mainly associated with neural activities of the motor areas and may better reflect regional motor neural activities. Although the exact mechanisms still need to be further exploited, complete understanding of the mechanism may involve long-term fine-tuning of the dominant motor cortex focusing on more frequent motor learning, skill acquisition, and motor practice for the dominant hand.

The motor cortex controls, modulates motor action, and vastly relies on a sensitive balance between cortical excitatory and inhibitory mechanisms. As examined in a recent study which focused on TMS navigation, it was discovered that the excitatory motor responses appeared to be significantly abundant on the dominant hemisphere than they were on the non-dominant hemisphere. It was found that the dominant hemisphere was better organized in controlling excitatory motor functions with respect to TMS [30]. The investigators explained that the results were possibly a consequence of long-term fine-tuning between cortical excitation and inhibition in the dominant hemisphere 
Citation: Li CT, Juan CH, Su TP, Liou YJ, Chen MH, et al. (2016) Transcranial Magnetic Stimulation-Derived Motor Parameters and Regional Glucose Metabolism of Motor Cortex. J Neurol Neurophysiol 7: 386. doi:10.4172/2155-9562.1000386
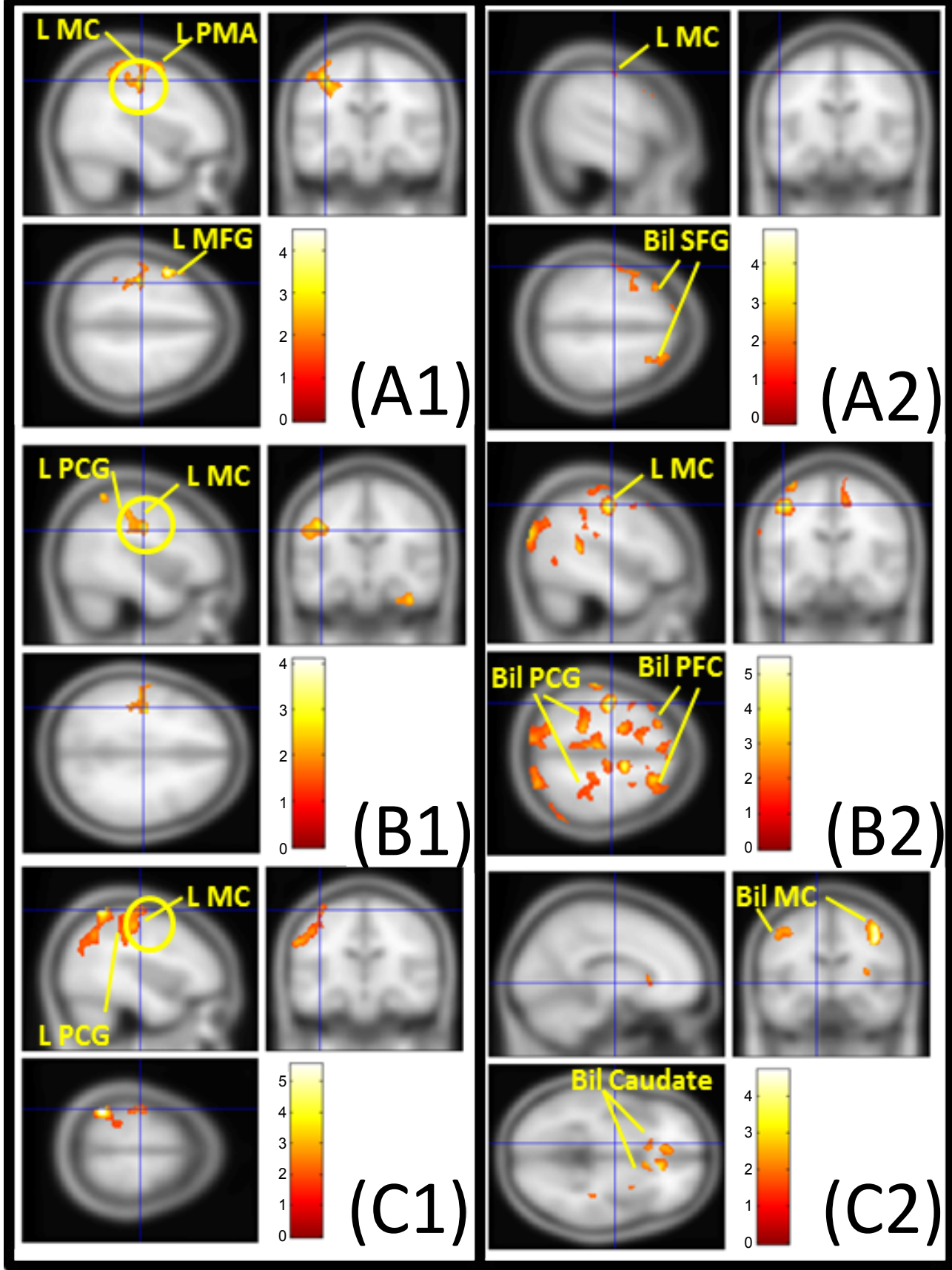

Figure 2: Voxel-wise correlations of TMS-derived parameters and regional glucose metabolism (rGluM). (A) Resting motor threshold; (B) Amplitude of motor evoked potentials; (C) Long-interval intra-cortical inhibition. The correlation results for TMS parameters from the dominant left-side hemisphere are shown in the left pane (A1-C1), while the results for TMS parameters from the non-dominant right-side hemisphere are shown in the right panel (A2-C2). The dominant motor cortex-derived TMS parameters, unlike those of the non-dominant side, were mainly associated with the measured motor areas (circled in yellow color) and may better reflect regional motor neural activities. Partial correlations of TMS parameters and rGluM were performed once controlling for age, gender, and whole-brain glucose uptake values. Statistically significant brain regions were drawn (family-wise errors-corrected p $<0.005$ ), and contrast bars denote t-values. L-Left; R-Right: MC, Motor Cortex; PMA, Premotor Area; MFG, Middle Frontal Gyrus; SFG, Superior Frontal Gyrus; PCG, Post-Central Gyrus (sensory cortex); PFC, Prefrontal Cortex; CG, Cingulate Gyrus; IFG, Inferior Frontal Gyrus.

which take place during the growth and motor learning process. Repetitive motor performance, skill learning, and even cognitive motor actions have also been known to reorganize brain function by using a plasticity mechanism [31,32].

Higher regional glucose metabolism in the dominant motor cortex may be the result of life-time asymmetry in the motor performance, which confirms findings from previous studies which demonstrated an increased neural complexity and fiber density of the dominant motor area $[33,34]$. It is however noteworthy that the dominantside amplitude of MEP showed negative correlation to the glucose metabolism of the dominant (left) motor cortex and that the dominantside $\mathrm{rMT}$ demonstrated a positive correlation to the glucose metabolism 
of the dominant (left) motor cortex. We can only speculate that since ${ }^{18}$ F-FDG-PET signals do not measure only the excitatory activity of neurons but also those of inhibitory neurons. It is a possibility that the negative correlation between the rGluM in the left motor cortex and the MEP size can be explained by the inhibitory activity of neurons. That is, higher activity of the inhibitory neurons in the dominant motor cortex explains the higher rGluM corresponding to smaller MEP size.

Previous research has indicated that muscle training was associated with reductions in ICI in both agonist and antagonist muscles [35]. A decrease in GABA-related inhibition facilitates practice-dependent plasticity in the human motor cortex [36]. McDonnel et al. further showed that $\mathrm{GABA}_{\mathrm{B}}$ receptor agonist baclofen decreases long-term potentiation-like plasticity in human motor cortex. This suggests that the motor learning process directly involves a reduction of $\mathrm{GABA}_{\mathrm{B}}$ mediated and not a $\mathrm{GABA}_{\mathrm{A}}$-mediated, inhibitory function [37]. The notion could be further supported by our finding where the dominant motor cortex showed a significantly weaker LICI, but higher regional glucose metabolism. It has also been found that $\mathrm{GABA}_{\mathrm{B}}$-mediated LICI could inhibit SICI through presynaptic $\mathrm{GABA}_{\mathrm{B}}$ receptors [38]. Therefore, the reduced LICI in the dominant motor cortex would consequently enhance activities of some inhibitory neurons via decreasing $\mathrm{GABA}_{\mathrm{B}}$-mediated inhibition. Long-term learning of highly skilled movement could likewise result in long-term change of plasticity of the motor cortex [31].

Meanwhile, both excitatory and inhibitory TMS-derived measurements from the non-dominant motor cortex were found to be connected to numerous regions outside the motor cortex, including but not limited to the motor cortex. For instance, right rMT is correlated with rGluM in some areas of the bilateral frontal cortices. Significant relationships were observed between right MEPs and rGluM in the postcentral gyri, middle occipital gyri, middle frontal gyri, superior temporal gyri, and middle cingulate gyri (Figure 2). Poor organization and lesser specificity of the non-dominant motor cortex of the corticospinal tract could possibly explain why there were more correlations found outside the motor cortex. Whether or not long-term motor practice on the non-dominant hand might change the plasticity warrants, is worth exploring in future studies. Although our study did not aim to investigate the correlations of TMS-derived parameters and brain regions outside the motor cortex, our findings are conclusive that the non-dominant TMS measurements may have more potential than those from the dominant side with respect to assessing areas outside of the motor circuit. This rationale may be the explanation for reported abnormal cortical excitability in the non-dominant instead of the dominant motor cortex in psychiatric disorders [10-12].

Limitations to this study included the study design. The experiment design may not have been ideal for examining correlations between single-pulse and or paired-pulse TMS measurements with neural activities in the brain because the two experiments were done separately. The aim of performing these two experiments separately aimed at reducing lengthy radiation risks, in doing so, may be have created limitations. We however made all efforts to perform the TMS experiments and the PET scans on the same day while participants maintained a fully relaxed state. Secondly, the TMS parameters and PET scans were measured at a resting state of participants. Considering the larger side-to-side variation in motor performance, further study examining the correlation of TMS parameters and the brain metabolism in PET scan while simultaneously performing motor tasks is worth exploring. Thirdly, the study only recruited right-handed healthy subjects. Recruiting left-handed participants could possibly lead to promising conclusions and is worthy to further investigate in future studies. Lastly, since the current study was limited to only the regional GABA and glutamate-mediated inhibition and excitatory functions, it is crucial to also further explore the non-global GABA and glutamatemediated techniques.

\section{Conclusion}

This study supports findings that there are variable TMS-derived parameters measured from the dominant and non-dominant motor cortices and their underlying neural activities Such results indicate that the dominant-side derived TMS parameters better reflect regional motor activities and are thus more appropriate for studying motor functions. Furthermore, our results suggest that the non-dominant-side parameters may be influenced by more widespread regions other than the motor cortex. This rationale can serve as a useful tool for evaluation functions located outside the motor system.

\section{Acknowledgement}

This study received grants from Taipei Veterans General Hospital (V103B-007, V103E9-005, V104E9-003, and V104B-002), the Ministry of Science and Technology (MOST 102-2314-B-182-030-MY3 and 103-2314-B-075-072-MY3), the National Health Research Institutes of Taiwan (NHRI-EX103-10343NI), Chang Gung Memorial Hospital (BMRP844), and the Military Medical Bureau (DV103-12). None of the aforementioned organizations had any role in the study design, data collection, analysis, interpretation of results, writing of the report, or the ultimate decision to submit the paper for publication.

\section{References}

1. Barker AT, Jalinous R, Freeston IL (1985) Non-invasive magnetic stimulation of human motor cortex. Lancet 1: 1106-1107.

2. Badawy RA, Loetscher T, Macdonell RA, Brodtmann A (2012) Cortical excitability and neurology: Insights into the pathophysiology. Funct Neurol 27 131-145.

3. Bunse T, Wobrock T, Strube W, Padberg F, Palm U, et al. (2014) Motor cortical excitability assessed by transcranial magnetic stimulation in psychiatric disorders: A systematic review. Brain Stimul 7: 158-169.

4. Radhu N, de Jesus DR, Ravindran LN, Zanjani A, Fitzgerald PB, et al. (2013) A meta-analysis of cortical inhibition and excitability using transcranial magnetic stimulation in psychiatric disorders. Clin Neurophysiol 124: 1309-1320.

5. Greenberg BD, Ziemann U, Cora-Locatelli G, Harmon A, Murphy DL, et al. (2000) Altered cortical excitability in obsessive-compulsive disorder. Neurology 54: 142-147.

6. Grunhaus L, Polak D, Amiaz R, Dannon PN (2003) Motor-evoked potential amplitudes elicited by transcranial magnetic stimulation do not differentiate between patients and normal controls. Int J Neuropsychopharmacol 6: 371 378

7. Levinson AJ, Fitzgerald PB, Favalli G, Blumberger DM, Daigle M, et al. (2010) Evidence of cortical inhibitory deficits in major depressive disorder. Biol Psychiatry 67: 458-464.

8. Reid PD, Daniels B, Rybak M, Turnier-Shea Y, Pridmore S (2002) Cortica excitability of psychiatric disorders: Reduced post-exercise facilitation in depression compared to schizophrenia and controls. Aust N Z J Psychiatry 36: 669-673.

9. Richter MA, de Jesus DR, Hoppenbrouwers S, Daigle M, Deluce J, et al (2012) Evidence for cortical inhibitory and excitatory dysfunction in obsessive compulsive disorder. Neuropsychopharmacology 37: 1144-1151.

10. Bajbouj M, Lisanby SH, Lang UE, Danker-Hopfe H, Heuser I, et al. (2006) Evidence for impaired cortical inhibition in patients with unipolar major depression. Biol Psychiatry 59: 395-400.

11. Maeda F, Keenan JP, Pascual-Leone A (2000) Interhemispheric asymmetry of motor cortical excitability in major depression as measured by transcranial magnetic stimulation. Br J Psychiatry 177: 169-173.

12. Rossi S, De Capua A, Tavanti M, Calossi S, Polizzotto NR, et al. (2009) Dysfunctions of cortical excitability in drug-naive posttraumatic stress disorder patients. Biol Psychiatry 66: 54-61. 
Citation: Li CT, Juan CH, Su TP, Liou YJ, Chen MH, et al. (2016) Transcranial Magnetic Stimulation-Derived Motor Parameters and Regional Glucose Metabolism of Motor Cortex. J Neurol Neurophysiol 7: 386. doi:10.4172/2155-9562.1000386

13. Pallanti S, Borgheresi A, Pampaloni I, Giovannelli F, Bernardi S, et al. (2010) Motor cortex excitability correlates with novelty seeking in social anxiety: A transcranial magnetic stimulation investigation. J Neurol 257: 1362-1368.

14. Rossi S, Hallett M, Rossini PM, Pascual-Leone A (2009) Safety, ethical considerations, and application guidelines for the use of transcranial magnetic stimulation in clinical practice and research. Clin Neurophysiol 120: 2008-2039.

15. Liepert J, Schwenkreis P, Tegenthoff M, Malin JP (1997) The glutamate antagonist riluzole suppresses intracortical facilitation. J Neural Transm 104: 1207-1214.

16. Ziemann U, Lönnecker S, Steinhoff BJ, Paulus W (1996) The effect of lorazepam on the motor cortical excitability in man. Exp Brain Res 109: 127-135.

17. Ziemann U, Lönnecker S, Steinhoff BJ, Paulus W (1996b) Effects of antiepileptic drugs on motor cortex excitability in humans: A transcranial magnetic stimulation study. Ann Neurol 40: 367-378.

18. Sibson NR, Dhankhar A, Mason GF, Rothman DL, Behar KL, et al. (1998) Stoichiometric coupling of brain glucose metabolism and glutamatergic neuronal activity. Proc Natl Acad Sci U S A 95: 316-321.

19. Carlson PJ, Diazgranados N, Nugent AC, Ibrahim L, Luckenbaugh DA, et al. (2013) Neural correlates of rapid antidepressant response to ketamine in treatment-resistant unipolar depression: A preliminary positron emission tomography study. Biol Psychiatry 73: 1213-1221.

20. Li CT, Chen MH, Lin WC, Hong CJ, Yang BH, et al. (2016) The effects of lowdose ketamine on the prefrontal cortex and amygdala in treatment-resistan depression: A randomized controlled study. Hum Brain Mapp 37: 1080-1090.

21. Marsteller DA, Barbarich-Marsteller NC, Patel VD, Dewey SL (2007) Brain metabolic changes following 4-week citalopram infusion: increased 18FDG uptake and gamma-amino butyric acid levels. Synapse 61: 877-881.

22. Oldfield RC (1971) The assessment and analysis of handedness: the Edinburgh inventory. Neuropsychologia 9: 97-113.

23. Kujirai T, Caramia MD, Rothwell JC, Day BL, Thompson PD, et al. (1993) Cortico-cortical inhibition in human motor cortex. J Physiol 471: 501-519.

24. Valls-Solé J, Pascual-Leone A, Wassermann EM, Hallett M (1992) Human moto evoked responses to paired transcranial magnetic stimuli. Electroencephalogr Clin Neurophysiol 85: 355-364.

25. Li CT, Hsieh JC, Wang SJ, Yang BH, Bai YM, et al. (2012) Differential relations between fronto-limbic metabolism and executive function in patients with remitted bipolar I and bipolar II disorder. Bipolar Disord 14: 831-842.
26. Hong R, Halama J, Bova D, Sethi A, Emami B (2007) Correlation of PET standard uptake value and CT window-level thresholds for target delineation in CT-based radiation treatment planning. Int J Radiat Oncol Biol Phys 67: 720726.

27. Gispert JD, Pascau J, Reig S, Martínez-Lázaro R, Molina V, et al. (2003) Influence of the normalization template on the outcome of statistical parametric mapping of PET scans. Neuroimage 19: 601-612.

28. Signorini M, Paulesu E, Friston K, Perani D, Colleluori A, et al. (1999) Rapid assessment of regional cerebral metabolic abnormalities in single subjects with quantitative and nonquantitative [18F] FDG PET: A clinical validation of statistical parametric mapping. Neuroimage 9: 63-80.

29. Hirvonen J, Goodwin RS, Li CT, Terry GE, Zoghbi SS, et al. (2012) Reversible and regionally selective down regulation of brain cannabinoid CB1 receptors in chronic daily cannabis smokers. Mol Psychiatry 17: 642-349.

30. Pitkänen M, Kallioniemi E, Julkunen P (2015) Extent and location of the excitatory and inhibitory cortical hand representation maps: A navigated transcranial magnetic stimulation study. Brain Topogr 28:657-665.

31. Ljubisavljevic M (2006) Transcranial magnetic stimulation and the motor learning-associated cortical plasticity. Exp Brain Res 173: 215-222.

32. Sanes JN, Donoghue JP (2000) Plasticity and primary motor cortex. Annu Rev Neurosci 23: 393-415.

33. Amunts K, Schlaug G, Schleicher A, Steinmetz H, Dabringhaus A, et al. (1996) Asymmetry in the human motor cortex and handedness. Neuroimage 4: 216222

34. Büchel C, Raedler T, Sommer M, Sach M, Weiller C, et al. (2004) White matter asymmetry in the human brain: A diffusion tensor MRI study. Cereb Cortex 14 945-951.

35. Classen J, Liepert J, Hallett M, Cohen L (1999) Plasticity of movement representation in the human motor cortex. Electroencephalogr Clin Neurophysiol Suppl 51: 162-173.

36. Ziemann U, Muellbacher W, Hallett M, Cohen LG (2001) Modulation of practicedependent plasticity in human motor cortex. Brain 124: 1171-1181.

37. McDonnell MN, Orekhov Y, Ziemann U (2007) Suppression of LTP-like plasticity in human motor cortex by the GABAB receptor agonist baclofen. Exp Brain Res 180: 181-186.

38. Sanger TD, Garg RR, Chen R (2001) Interactions between two different inhibitory systems in the human motor cortex. J Physiol 530: 307-317. 